\title{
Sound focusing by gradient index sonic lenses
}

\author{
Alfonso Climente, Daniel Torrent, and José Sánchez-Dehesa ${ }^{\text {a) }}$ \\ Grupo de Fenómenos Ondulatorios, Departamento de Ingeniería Electrónica, Universidad Politécnica de \\ Valencia, Camino de Vera s.n., E-46022 Valencia, Spain
}

(Received 11 June 2010; accepted 18 August 2010; published online 8 September 2010)

\begin{abstract}
Gradient index sonic lenses based on two-dimensional sonic crystals are here designed, fabricated, and characterized. The index-gradient is achieved in these type of flat lenses by a gradual modification of the sonic crystal filling fraction along the direction perpendicular to the lens axis. The focusing performance is well described by an analytical model based on ray theory as well as by numerical simulations based on the multiple-scattering theory. (C) 2010 American Institute of Physics. [doi:10.1063/1.3488349]
\end{abstract}

A two-dimensional (2D) sonic crystal (SC) is just a periodic distribution of solid cylinders in air with their axis parallel aligned. The properties of these structures have been already analyzed in a broad range of frequencies. Thus, for frequencies of the order of the lattice separation they present a band of frequencies (band gap) in which the sound propagation is forbidden because of Bragg reflection. ${ }^{1}$ However, in the range of low frequencies (homogenization limit) they behave like homogeneous media whose effective acoustic parameters, dynamical mass density, and bulk modulus, basically depend on the lattice filling fraction. ${ }^{2-4}$

The homogenization properties of SCs have been employed to design refractive devices like, for example, acoustic lenses whose focusing properties are based on their external curved surfaces ${ }^{5-7}$ or Fabry-Perot type acoustic interferometers. ${ }^{8}$ Gradient index (GRIN) sonic lenses based on homogenized 2D SCs have been proposed. ${ }^{9}$ The index gradient was obtained by changing the SC filling fraction, which is directly related to the local refraction index. 2D GRIN SCs composed of different cylinder materials along different rows have been also theoretically demonstrated. ${ }^{10}$ Like their optical counterparts, the proposed 2D GRIN SC lenses have flat surfaces and are of easier fabrication than curved SC lenses. More recently, a GRIN SC lens based on a different approach has been proposed and experimentally demonstrated $;{ }^{11}$ it is designed for a frequency within the first acoustic band with negative slope. The drawback of this type of GRIN lenses, whose wavelengths are of the order of the lattice parameter, is that they work for the single operational frequency at which they have been designed. The bending of sound waves obtained by GRIN acoustics structures have been also proposed to produce acoustic mirages in a laboratory. $^{12}$

In this paper 2D GRIN SC lenses have been fabricated and characterized by an specifically designed experimental set up. Each lens is made of a rectangular cluster of aluminum cylinders distributed in a hexagonal lattice. Their focusing properties are here comprehensively studied either experimentally as well as theoretically. We demonstrate that their focusing behavior is a true GRIN effect that is fairly well reproduced by simulations based in the multiple scattering theory (MST) and it is also supported by an analytical model based on ray theory.

\footnotetext{
${ }^{a)}$ Electronic mail: jsdehesa@upvnet.upv.es.
}

The acoustical axis of GRIN lenses considered here is defined along the $x$-axis and, therefore, the refractive index $n(y)$ changes along the $y$-axis. The local variation in $n$ is obtained by changing the cylinders radii as explained in Ref. 9. A GRIN lens and the region of data acquisition are schematically shown in Fig. 1.

The index profile $n(y)$ is designed with the goal of having low aberration of the focal spot. We choose a hyperbolic secant profile because of its demonstrated property of focusing sound inside large enough GRIN structures without aberration: ${ }^{10}$

$$
n(y)=n_{0} \operatorname{sech}(\alpha y),
$$

where $\alpha$ is a constant defined as

$$
\alpha=\frac{1}{h} \cosh ^{-1}\left(\frac{n_{0}}{n_{h}}\right),
$$

where $h$ is the half-height of the lens, $n_{0}$ is the refractive index on the $x$-axis $(y=0)$ and $n_{h}$ that at the lens edges $(y= \pm \mathrm{h})$.

The differential equation satisfied by the energy rays can be obtained ${ }^{13}$ and by solving it, the position of the focal spot outside the lens, $x_{f}$, is obtained

$$
x_{f}=d-\frac{y(d)}{y^{\prime}(d)} \sqrt{\frac{1-\left[y^{\prime}(d)\right]^{2}\left\{n^{2}[y(d)]-1\right\}}{n^{2}[y(d)]}},
$$

where $d$ is the lens thickness.

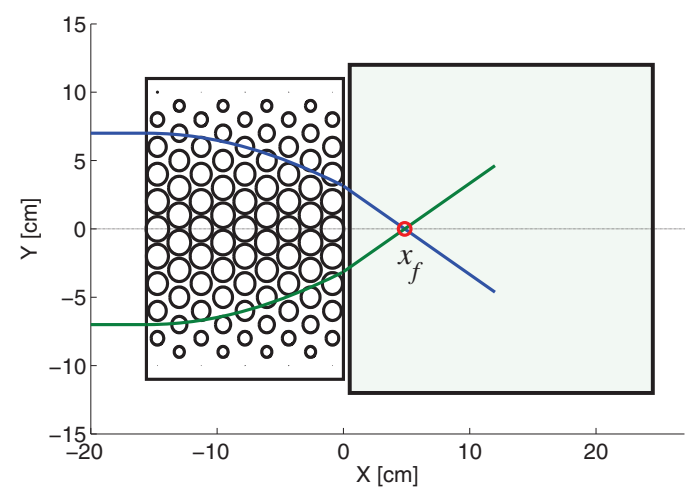

FIG. 1. (Color online) Schematic view of a GRIN lens made of nine columns of metal rods. Circles define the rods sections, which are position dependent. The sound impinging the lens from the left bends toward the $x$ axis and focuses at position $x_{f}$ (circle). The gray rectangle defines the area where the data are acquired. 
TABLE I. Effective refractive index profile $n_{\text {eff }}(y)$ of the 2D GRIN SC lenses studied here.

\begin{tabular}{|c|c|c|c|c|c|c|}
\hline \multirow[b]{2}{*}{$\begin{array}{c}y_{\ell} \\
(\mathrm{mm})\end{array}$} & \multirow[b]{2}{*}{$n\left(y_{\ell}\right)$} & \multirow[b]{2}{*}{$n_{\text {eff }}\left(y_{\ell}\right)$} & \multicolumn{2}{|c|}{ Odd layers } & \multicolumn{2}{|c|}{ Even layers } \\
\hline & & & $\ell$ & $\begin{array}{c}R_{\ell} \\
(\mathrm{mm})\end{array}$ & $\ell$ & $\begin{array}{c}R_{\ell} \\
(\mathrm{mm})\end{array}$ \\
\hline-100 & 1 & 1 & 1 & 0 & $\ldots$ & $\ldots$ \\
\hline-90 & 1.052 & 1.070 & $\ldots$ & $\ldots$ & 1 & 8.0 \\
\hline-80 & 1.102 & 1.107 & 2 & 10.0 & $\cdots$ & $\cdots$ \\
\hline-70 & 1.149 & 1.151 & $\cdots$ & $\ldots$ & 2 & 12.0 \\
\hline-60 & 1.193 & 1.201 & 3 & 14.0 & $\cdots$ & $\cdots$ \\
\hline-50 & 1.232 & 1.228 & $\cdots$ & $\cdots$ & 3 & 15.0 \\
\hline-40 & 1.266 & 1.258 & 4 & 16.0 & $\cdots$ & $\cdots$ \\
\hline-30 & 1.293 & 1.293 & $\cdots$ & $\ldots$ & 4 & 17.0 \\
\hline-20 & 1.314 & 1.313 & 5 & 17.5 & & $\cdots$ \\
\hline-10 & 1.326 & 1.339 & $\cdots$ & $\cdots$ & 5 & 18.0 \\
\hline 0 & 1.330 & 1.339 & 6 & 18.0 & $\cdots$ & $\cdots$ \\
\hline
\end{tabular}

A 2D GRIN SC can be considered as a homogenized medium made of subwavelength discrete units. The units are metal cylinders that are distributed in rows of equal radii. A given row $\ell$ is parallel to the $x$-axis and it is independently homogeneous and isotropic. The refractive index at row with coordinate $y_{\ell}$ is adjusted by taking into account that, in the low frequency limit, a cluster of rigid cylinders embedded in a fluid or gas background behaves like a homogeneous medium whose effective refractive index $n_{\text {eff }}$ mainly depends on the fraction of volume occupied by the cylinder in the SC. 3.4

The 2D GRIN SC lenses studied are designed with a profile $n(y)$ as closer as possible to that in Eq. (1) with $n_{0}$ $=1.337$ and $n_{h}=1$. Each lens is made of a rectangular cluster of aluminum cylinders distributed in an hexagonal lattice with lattice parameter $a=2 \mathrm{~cm}$ (see Fig. 1). The radii $R_{\ell}$ of cylinders at row $y_{\ell}$ is obtained by imposing the condition $n_{\text {eff }}(f)=n\left(y_{\ell}\right)$, where $f=2 \pi / \sqrt{3}\left(R_{\ell} / a\right)^{2}$ is the filling fraction of the hexagonal lattice. Table I reports the values of $R_{\ell}$ obtained. Note that values corresponding to the lower half of the SC lens are the only ones reported since the lens is symmetric with respect to the $x$-axis. Odd layers have 11 cylinders while even layers have 10 cylinders. Column $n\left(y_{\ell}\right)$ in table report the exact hyperbolic secant profile while those under $n_{\text {eff }}\left(y_{\ell}\right)$ are those effectively achieved in samples.

The lattice spacing $a$ determines an upper frequency limit for the SC homogenization. It has been demonstrated that the homogenization of a SC takes place for wavelengths larger than a certain cutoff given approximately by $\lambda_{c} \approx 4 a{ }^{3}$ Since our fabricated GRIN lenses are based on hexagonal lattices with $a=2 \mathrm{~cm}$, their focusing properties will appear at wavelengths $\lambda \geq 8 \mathrm{~cm}$, which corresponds to linear frequencies $\nu \leq 4.3 \mathrm{kHz}$. However, let us stress that the onset for the homogenization is not an exact parameter and we report below GRIN lenses working at $4.5 \mathrm{kHz}$, which is a frequency slightly above the cutoff $\nu_{c}=4.3 \mathrm{kHz}$. Moreover, it will be shown that a better agreement with the ray model of Eq. (3) is obtained in a wider range of lens thicknesses for frequencies near $\nu_{c}$.

The lenses have been characterized in a 2D anechoic chamber specifically designed for the parameters of the lenses. The chamber consists of two parallel metal sheets separated a distance $H=5 \mathrm{~cm}$. For the frequencies analyzed here, from $3.5 \mathrm{kHz}$ up to $4.5 \mathrm{kHz}$, there are two modes propagating trough the chamber, but the second one has a much larger wavelength and is considered as an offset. The sound waves are generated with a UDE AC-150 column loudspeaker separated $2 \mathrm{~m}$ from the chamber in order to get approximately a plane wavefront at the entrance. The input signal is a Gaussian pulse centered at the frequencies in between the range under study. A total of eleven frequencies are comprehensively analyzed; i.e., from $3.5 \mathrm{kHz}$ till $4.5 \mathrm{kHz}$ in steps of $0.1 \mathrm{kHz}$. Measurements made without lens confirm that the propagating field inside the chamber has practically a plane wavefront. We use a B\&K 4958 microphone to record the transmitted sound behind the lens and use the PCI Acquisition Card NI-5105 for processing the data in a computer. Data are acquired in a square area of 24 $\times 24 \mathrm{~cm}^{2}$, which is represented in Fig. 1. The acquisition points are separated by equal distance along the $x$ - and $y$-axis; i.e., $\Delta x=\Delta y=2 \mathrm{~cm}$.

Seven samples have been experimentally characterized. The lenses differ in the number $N$ of layers defining their thickness along the $x$-axis; from 4 to 10 . The corresponding pressure maps have been measured and compared with those obtained by numerical simulations based on the MST. Besides, the focal spots $x_{f}$ have been also located and their values are compared with those predicted by Eq. (3). Measurements and simulations are performed in the range of frequencies explored every $0.1 \mathrm{kHz}$.

Numerical simulations are performed by using a 2D multiple scattering algorithm as described in Refs. 7 and 8, where the reader is addressed for details. In brief, a 2D sound wave with a plane wavefront impinges the lens from the left and the pressure map is calculated in the opposite side.

As a typical example of results obtained, Figs. 2(a) and 2(b) show the sound amplification maps for a nine layer thick GRIN lens for the frequency of $4.5 \mathrm{kHz}$; their corresponding wavelength, $\lambda \approx 3.8 a$, roughly corresponds to the cutoff defining the homogenization limit, The focusing effects, focal spot and diffraction lobes, are clearly seen in both maps, measured [see Fig. 2(a)] and calculated [see Fig. 2(b)]. A better comparison between data and simulations is given in Figs. 3(a) and 3(b) that show the longitudinal and transversal profiles passing through the focal spot obtained from the previous pressure maps. Note that a fairly good agreement exists between data and simulations. A similar agreement is also obtained for the other frequencies analyzed.

In spite of the agreement reported above between theory and experiment a comparison with the position of the focal spot $x_{f}$ predicted by the ray theory of Eqs. (1)-(3) has been also performed in order to support that sound focusing is a truly refractive effect produced by the bending of waves inside the GRIN lens. The parameters employed in Eq. (3) are determined as follows. The lens half-height $h$ is obtained from $2 h=N_{y} a$ where $N_{y}$ is the maximum number of cylinders along the $y$-axis, $N_{y}=11$. As index profile, we used the ideal values $n\left(y_{\ell}\right)$ in Table I. The lens thickness $d$, which is the factor leading the discrepancy between the ray method and measurement/simulation results, is obtained from $d$ $=N(\sqrt{3} / 2) a+k$, where $N$ is the number of cylinders' layers employed in building the lens and $k$ is an adjustable parameter used to fit $x_{f}$ to measured data. The values obtained are $k=-0.41 \mathrm{~cm}$ for $3.5 \mathrm{kHz}$, and $k=-0.28 \mathrm{~cm}$ for $4.5 \mathrm{kHz}$, which means that the lens surface is nearer to the lens units than that usually employed in solid state physics, ${ }^{14}$ where the surface position is taken at a distance above the atoms equal to a half of the lattice parameter; i.e., $k=0$ in our model. 

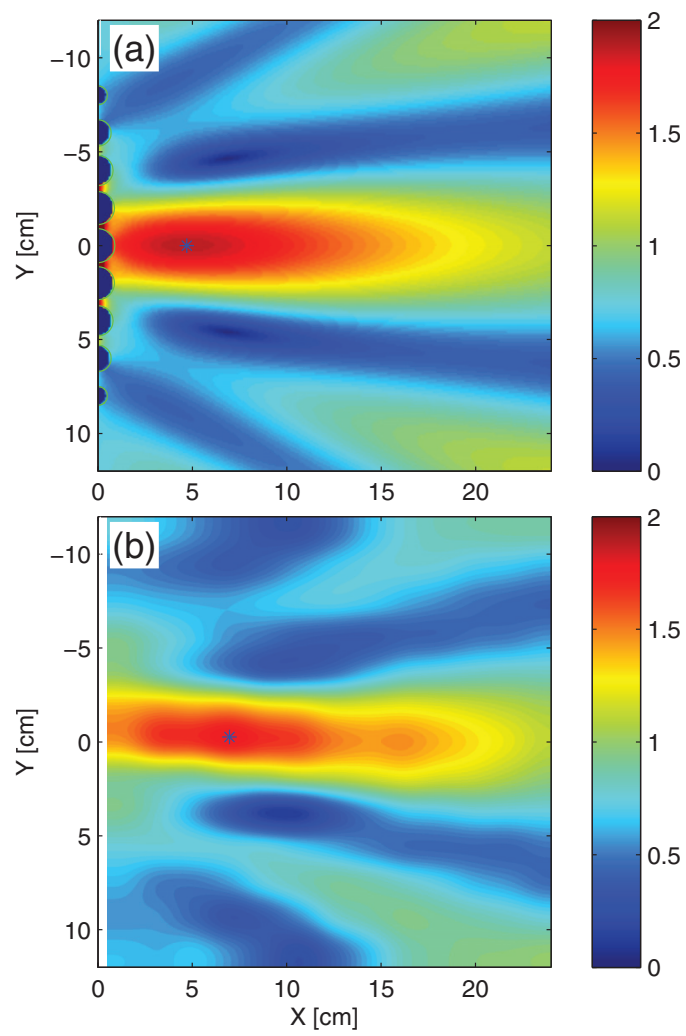

FIG. 2. (Color online) (a) Sound amplification map generated by $4.5 \mathrm{kHz}$ sound waves impinging a nine layers thick 2D GRIN SC lens. The map has been obtained by using an algorithm based on the multiple scattering method. (b) The corresponding map measured in our experimental set up. Both maps are obtained in an area $24 \times 24 \mathrm{~cm}^{2}$ near the lens (see Fig. 1). The asterisks mark the focal spot $x_{f}$.

Figure 4 reports results obtained for $x_{f}$ at the lower and higher frequencies analyzed: (a) $3.5 \mathrm{kHz}$ and (b) $4.5 \mathrm{kHz}$.

Data and multiple scattering simulations show a better agreement with the ray model when: (i) the frequency operation is near the homogenization limit and (ii) for the thicker lens. These conclusions are physically intuitive. On the one hand, for lower frequencies (larger wavelengths) the homogenization takes place over a larger number of unit cells in the lattice and, as a consequence, the locally designed refractive

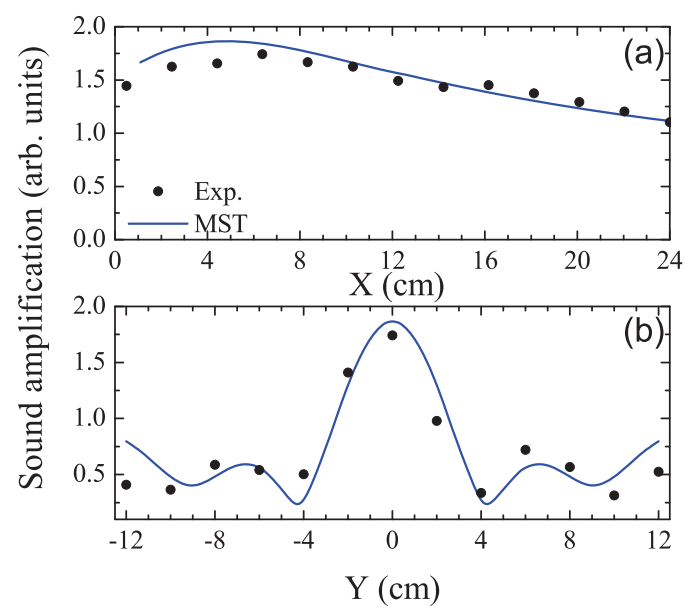

FIG. 3. (Color online) (a) Longitudinal and (b) transversal cuts extracted from the maps shown in Fig. 2. Simulations based on MST (continuous lines) and experimental data (black dots) are depicted.

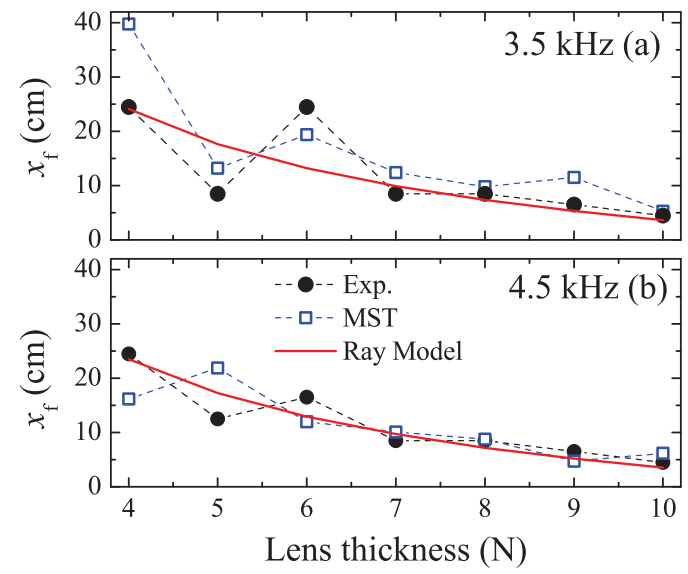

FIG. 4. (Color online) Focal spot, $x_{f}$, as a function of $N$, the number of layers defining the lens thickness, for frequencies: (a) $3.5 \mathrm{kHz}$ and (b) 4.5 $\mathrm{kHz}$. Black dots and hollow squares represent experimental data are results obtained from a MST, respectively. The dashed lines between symbols are guides for the eye. Continuous lines represent predictions obtained by a ray model.

index profile $n(y)$ could be partially destroyed. On the second hand, thicker lenses means that sound travels longer distance inside the lens and consequently the role of surfaces and diffraction effects become negligible.

In summary, we have demonstrated the focusing properties of 2D GRIN sonic lenses made of aluminum rods. Their broadband performance has been confirmed by numerical simulations based on a multiple scattering algorithm and by an analytical model based on ray theory. It can be concluded that GRIN sonic lenses are a type of refractive acoustical devices that are feasible and reliable for possible applications not only in the sonic range, as has been demonstrated here, but also for ultrasonics and even for acoustic surface waves as has been previously proposed.

Work partially supported by USA Office of Naval Research (Grant No. N000140910554) and by the Spanish MICIIN under Contract Nos. TEC2007-67239 and CSD2008-66 (CONSOLIDER program).

${ }^{1}$ J. V. Sánchez-Pérez, D. Caballero, R. Martinez-Sala, C. Rubio, J. SánchezDehesa, F. Meseguer, J. Llinares, and F. Gálvez, Phys. Rev. Lett. 80, 5325 (1998).

${ }^{2}$ A. A. Krokhin, J. Arriaga, and L. N. Gumen, Phys. Rev. Lett. 91, 264302 (2003).

${ }^{3}$ D. Torrent, A. Håkansson, F. Cervera, and J. Sánchez-Dehesa, Phys. Rev. Lett. 96, 204302 (2006).

${ }^{4}$ D. Torrent and J. Sánchez-Dehesa, Phys. Rev. B 74, 224305 (2006).

${ }^{5}$ E. Meyer and E. G. Neumann, Physical and Applied Acoustics (Academic, New York, 1972).

${ }^{6}$ F. Cervera, L. Sanchis, J. V. Sánchez-Pérez, R. Martinez-Sala, C. Rubio, F. Meseguer, C. López, D. Caballero, and J. Sánchez-Dehesa, Phys. Rev. Lett. 88, 023902 (2001).

${ }^{7}$ B. C. Gupta and Z. Ye, Phys. Rev. E 67, 036603 (2003).

${ }^{8}$ L. Sanchis, A. Håkansson, F. Cervera, and J. Sánchez-Dehesa, Phys. Rev. B 67, 035422 (2003).

${ }^{9}$ D. Torrent and J. Sánchez-Dehesa, New J. Phys. 9, 323 (2007).

${ }^{10}$ S.-C. S. Lin, T. J. Huang, J. H. Sun, and T. T. Wu, Phys. Rev. B 79, 094302 (2009)

${ }^{11}$ S. Peng, Z. He, H. Jia, A. Zhang, C. Qiu, M. Ke, and Z. Liu, Appl. Phys. Lett. 96, 263502 (2010).

${ }^{12}$ S.-C. S. Lin and T. J. Huang, J. Appl. Phys. 106, 053529 (2009).

${ }^{13}$ C. Gómez-Reino, M. V. Pérez, and C. Bao, Gradient-Index Optics: Fundamentals and Applications (Springer, New York, 2002).

${ }^{14}$ K. W.-K. Shung and Y. C. Tsai, Phys. Rev. B 48, 11265 (1993). 